Proceedings of the Institute of Mathematics and Mechanics,

National Academy of Sciences of Azerbaijan

Volume 47, Number 2, 2021, Pages 309-326

https://doi.org/10.30546/2409-4994.47.2.309

\title{
SOLVABILITY OF A BOUNDARY VALUE PROBLEM FOR A SECOND ORDER ELLIPTIC DIFFERENTIAL-OPERATOR EQUATION WITH A COMPLEX PARAMETER
}

\author{
BAHRAM A. ALIEV, VUGAR Z. KERIMOV, AND YAKOV S. YAKUBOV
}

\begin{abstract}
In a separable Hilbert space $H$, we study solvability of a boundary value problem for a second order elliptic differential-operator equation with a complex parameter. The parameter enters into the equation and boundary conditions. In addition to the complex parameter, the boundary conditions contain linear operators as well, one of which is unbounded. Application of the obtained abstract results to elliptic boundary value problems is given.
\end{abstract}

\section{Introduction}

In the paper, in a separable Hilbert space $H$, we consider the following boundary value problem:

$$
\begin{gathered}
L(\lambda) u:=\lambda u(x)-u^{\prime \prime}(x)+A u(x)=f(x), x \in(0,1), \\
L_{1}(\lambda) u:=\lambda u(0)-\alpha u^{\prime}(0)+B_{1} u(1)=f_{1}, \\
L_{2}(\lambda) u:=\lambda u(1)+\beta u^{\prime}(1)+B_{2} u(0)=f_{2},
\end{gathered}
$$

where $\lambda$ is a complex parameter; $A$ is a linear, closed operator with domain of definition dense everywhere in $H$ and with the resolvent decreasing as $|\lambda|^{-1}$ for sufficiently large $\lambda$ in some angles, containing a negative semi-axis; $\alpha$ and $\beta$ are non-zero complex numbers from the right-hand side of the complex plane; $B_{1}$ is a linear bounded operator in $H ; B_{2}$ is a linear unbounded operator in $H$, subordinated to the operator $A^{1 / 2}$ in some sense.

It is proved that under certain conditions, for sufficiently large $\lambda$ from some angle, containing a positive semi-axis, a theorem on the isomorphism between the solutions, belonging to $W_{p}^{2}((0,1) ; H(A), H)$, and the right-hand side of the boundary value problem $(1.1),(1.2)$ is satisfied. It is also established the coercive estimate for the solution of the problem (1.1), (1.2) in the space $L_{p}((0,1) ; H), p \in$ $(1, \infty)$. More precisely, sufficient conditions, providing coercive solvability of the boundary value problem $(1.1),(1.2)$ with respect to $u$ in the space $L_{p}((0,1) ; H)$, $p \in(1, \infty)$, were found.

Note that if the boundary conditions in (1.2) do not contain the operators $B_{1}$ and $B_{2}$, the coercive solvability of the boundary value problem (1.1), (1.2) was

2010 Mathematics Subject Classification. 34G10, 34B40, 35J25.

Key words and phrases. Hilbert space, differential-operator equation, Fourier transform, interpolation spaces, isomorphism, elliptic equation. 
studied in [5] and [14] in the spaces $L_{p}((0,1) ; H)$ and $L_{p}((0,1) ; E), p \in(1, \infty)$, respectively. Here $E$ is a UMD Banach space (UMD -Unconditional Martingale Differences, whose definition is given, e.g., in monograph [9]).

The novelty of the present paper, unlike the papers [5], [14], is that the main part of the boundary value problem $(1.1),(1,2)$, in addition to a complex parameter, contains in the boundary conditions linear operators as well, one of which is unbounded.

If the boundary conditions in (1.2) do not contain a complex parameter $\lambda$ and the operator $B_{1}$ is a zero operator, the solvability of the obtained boundary value problem was studied in [2] and [6] in the spaces $L_{p}((0,1) ; H)$ and $L_{p}((0,1) ; E)$, respectively.

In [4], in the space $L_{p}((0,1) ; H), p \in(1, \infty)$, a non-coerciveness of a boundary value problem, with respect to $u$ and $\lambda$, for the equation (1.1) was studied. In [4], the principal part of one of the boundary conditions, in addition to the complex parameter $\lambda$, contains also a linear bounded operator as a multiplier.

Boundary value problems for second order elliptic differential-operator equations, when one (and the same) complex parameter is included into the equation and boundary conditions, were studied in different aspects in the papers [1], [3], [7], [8], [11] and others.

In the present paper, we will use the technique developed in monograph [13, chapter 5, section 5.4]. There, in $L_{p}((0,1) ; H), p \in(1, \infty)$, coercive solvability of boundary value problems was studied for a second order elliptic differentialoperator equation with nonlocal boundary conditions, when the coefficients in the boundary conditions are complex numbers. Solvability of a boundary value problem, considered in [13, chapter 5, section 5.4], was further studied in the paper [10] in the space $L_{p}((0,1) ; E), p \in(1, \infty)$.

The above mentioned novelty of the paper allows as to study solvability of a new class of boundary value problems for second order partial elliptic differential equations in nonsmooth domains. We give one of such applications for elliptic equations in the square at the end of the paper.

We introduce definitions and notions used in the paper. Let $E_{1}$ and $E_{2}$ be Banach spaces. The set $E_{1} \dot{+} E_{2}$ of all vectors of the from $(u, v)$, where $u \in E_{1}$, $v \in E_{2}$, with ordinary coordinate-linear operations and with the norm

$$
\|(u, v)\|_{E_{1} \dot{+} E_{2}}:=\|u\|_{E_{1}}+\|v\|_{E_{2}},
$$

is a Banach space and is called a direct sum of Banach spaces $E_{1}$ and $E_{2}$.

Let $E_{1}$ and $E$ be Banach spaces. By $B\left(E_{1}, E\right)$ we denote a Banach space of all linear bounded operators acting from $E_{1}$ into $E$, with ordinary operator norm. In a particular case, we assume $B(E):=B(E, E)$.

Let $A$ be a linear closed operator in $E$. Then, the domain of definition $D\left(A^{n}\right)$ of the operator $A^{n}$ turns into the Banach space $E\left(A^{n}\right)$ with respect to the norm

$$
\|u\|_{E\left(A^{n}\right)}:=\left(\sum_{k=0}^{n}\left\|A^{k} u\right\|_{E}^{2}\right)^{1 / 2} .
$$

The operator $A^{n}$ is bounded from $E\left(A^{n}\right)$ into $E$. In particular, if the operator $A$ has a bounded inverse in $E$, then

$$
\|u\|_{E\left(A^{n}\right)}:=\left\|A^{n} u\right\|_{E} .
$$


Let $E_{0}$ and $E_{1}$ be two Banach spaces continuously imbedded into the Banach space $E: E_{0} \subset E, E_{1} \subset E$. Such two spaces are called an interpolation pair $\left\{E_{0}, E_{1}\right\}$. Let us consider a Banach space (see [12], section 1.2.1):

$$
\begin{aligned}
& E_{0}+E_{1}:=\left\{u: u \in E, \exists u_{j} \in E_{j}, j=0,1, \text { where } u=u_{0}+u_{1},\right. \\
& \left.\|u\|_{E_{0}+E_{1}}=\inf _{\substack{u=u_{0}+u_{1} \\
u_{j} \in E_{j}}}\left(\left\|u_{0}\right\|_{E_{0}}+\left\|u_{1}\right\|_{E_{1}}\right)\right\} .
\end{aligned}
$$

According to the statement [12, section 1.3.1], the functional

$$
K(t, u):=\inf _{\substack{u=u_{0}+u_{1} \\ u_{j} \in E_{j}}}\left(\left\|u_{0}\right\|_{E_{0}}+t\left\|u_{1}\right\|_{E_{1}}\right)
$$

is continuous on $(0, \infty)$ with respect to $t$ and the following estimate holds

$$
\min \{1, t\}\|u\|_{E_{0}+E_{1}} \leq K(t, u) \leq \max \{1, t\}\|u\|_{E_{0}+E_{1}} .
$$

The interpolation space for $\left\{E_{0}, E_{1}\right\}$ is determined by the $K$-method as follows

$$
\begin{gathered}
\left(E_{0}, E_{1}\right)_{\theta, p}:=\left\{u: u \in E_{0}+E_{1},\|u\|_{\left(E_{0}+E_{1}\right)_{\theta, p}}:=\right. \\
\left.=\left(\int_{0}^{+\infty} t^{-1-\theta p} K^{p}(t, u) d t\right)^{1 / p}<\infty\right\}, 0<\theta<1, \quad 1 \leq p<\infty .
\end{gathered}
$$

Definition 1.1. [12, theorema 1.14.5] Let the operator $A$ generate an analytic, for $t>0$, and a strongly continuous, for $t \geq 0$, semigroup $e^{-t A}$ and this semigroup exponentially decreases. Then, the interpolation spaces $\left(E\left(A^{n}\right), E\right)_{\theta, p}$ of Banach spaces $E\left(A^{n}\right)$ and $E$ are determined by the equalities

$$
\begin{gathered}
\left(E\left(A^{n}\right), E\right)_{\theta, p}:=\left\{u: u \in E_{0}+E_{1},\|u\|_{\left(E\left(A^{n}, E\right)_{\theta, p}\right.}:=\right. \\
\left.=\left(\int_{0}^{+\infty} t^{-1+n \theta p}\left\|A^{n} e^{-t A} u\right\|_{E}^{p} d t\right)^{1 / p}<\infty\right\}, 0<\theta<1, \quad p \geq 1, n \in N .
\end{gathered}
$$

Moreover, by definition $\left(E\left(A^{n}\right), E\right)_{0, p}:=E\left(A^{n}\right), \quad\left(E\left(A^{n}, E\right)_{1, p}:=E\right.$.

Theorem 1.1. (interpolation theorem) [12, theorem $1.3 .3 /(a)]$ (see, also, $\left[13\right.$, section 1.7.9]). Let $\left\{E_{0}, E_{1}\right\}$ and $\left\{F_{0}, F_{1}\right\}$ be interpolation pairs. Denote by $B\left(\left\{E_{0}, E_{1}\right\},\left\{F_{0}, F_{1}\right\}\right)$ the set of linear operators mapping $E_{0}+E_{1}$ into $F_{0}+F_{1}$ and such that their contraction on $E_{k}, k=0,1$, continuously maps $E_{k}$ into $F_{k}$.

Then, for any $T \in B\left(\left\{E_{0}, E_{1}\right\},\left\{F_{0}, F_{1}\right\}\right)$, we have the inequality

$$
\|T\|_{B\left(\left(E_{0}, E_{1}\right)_{\theta, p},\left(F_{0}, F_{1}\right)_{\theta, p}\right)} \leq\|T\|_{B\left(E_{0}, F_{0}\right)}^{1-\theta}\|T\|_{B\left(E_{1}, F_{1}\right)}^{\theta}, 0<\theta<1, \quad 1 \leq p<\infty .
$$

Denote by $L_{p}((0,1) ; H), \quad 1<p<\infty$, a Banach space (for $p=2$, a Hilbert space) of vector-functions $x \rightarrow u(x):[0,1] \rightarrow H$ strongly measurable and summable in the $p$-th degree with the norm

$$
\|u\|_{L_{p}((0,1) ; H)}:=\left(\int_{0}^{1}\|u(x)\|_{H}^{p} d x\right)^{1 / p}<\infty .
$$


Denote also by $W_{p}^{2 n}\left((0,1) ; H\left(A^{n}\right), H\right):=\left\{u: A^{n} u, u^{(2 n)} \in L_{p}((0,1) ; H)\right\}$ a space of vector-functions with the norm

$$
\|u\|_{W_{p}^{2 n}\left((0,1) ; H\left(A^{n}\right), H\right)}:=\left\|A^{n} u\right\|_{L_{p}((0,1) ; H)}+\left\|u^{(2 n)}\right\|_{L_{p}((0,1) ; H)} .
$$

It is known [12, theorem 1.8.2] that if $u \in W_{p}^{2 n}\left((0,1) ; H\left(A^{n}\right), H\right)$ then

$$
u^{(j)}(\cdot) \in\left(H\left(A^{n}\right), H\right)_{\frac{j+1 / p}{2 n}, p}, \quad j=0, \ldots, 2 n-1 .
$$

\section{Homogeneous equation}

At first, in a separable Hilbert space $H$, we consider the following boundary value problem:

$$
\begin{gathered}
L(\lambda) u:=\lambda u(x)-u^{\prime \prime}(x)+A u(x)=0, x \in(0,1), \\
L_{1}(\lambda) u:=\lambda u(0)-\alpha u^{\prime}(0)+B_{1} u(1)=f_{1}, \\
L_{2}(\lambda) u:=\lambda u(1)+\beta u^{\prime}(1)+B_{2} u(0)=f_{2} .
\end{gathered}
$$

Theorem 2.1. Let the following conditions be fulfilled:

1) $A$ is a closed densely defined and invertible operator in the Hilbert space $H$ and

$$
\|R(\lambda, A)\| \leq C(1+|\lambda|)^{-1} \text { for }|\arg \lambda| \geq \pi-\varphi,
$$

where $R(\lambda, A):=(\lambda I-A)^{-1}$ is the resolvent of the operator $A$ and $\varphi \in(0, \pi)$ is some number;

2) $\alpha$ and $\beta$ are non-zero complex numbers with

$$
|\arg \alpha| \leq \frac{\pi-\varphi}{2} \text { and }|\arg \beta| \leq \frac{\pi-\varphi}{2}
$$

3) A linear operator $B_{1}$ is bounded from $H$ into $H$ and from $H(A)$ into $H(A)$;

4) $A$ linear closed operator $B_{2}$ is bounded from $H\left(A^{1 / 2}\right)$ into $H$ and from $H(A)$ into $H\left(A^{1 / 2}\right)$.

Then, for $f_{k} \in(H(A), H)_{\frac{1}{2}+\frac{1}{2 p}, p}, \quad p \in(1, \infty)$, and for sufficiently large $\lambda$ from the angle $|\arg \lambda| \leq \varphi-\varepsilon$, where $\varepsilon>0$ is a sufficiently small number, the problem (2.1), (2.2) has a unique solution $u(x) \in W_{p}^{2}((0,1) ; H(A), H)$ such that $u(0) \in D\left(B_{2}\right)$. Moreover, for such $\lambda$, for the solution we have the following estimate

$$
\begin{aligned}
& |\lambda|\|u\|_{L_{p}((0,1) ; H)}+\left\|u^{\prime \prime}\right\|_{L_{p}((0,1) ; H)}+\|A u\|_{\left.L_{p}(0,1) ; H\right)} \leq \\
& \leq C(\varphi, \varepsilon) \sum_{k=1}^{2}\left(\left\|f_{k}\right\|_{(H(A), H)_{\frac{1}{2}+\frac{1}{2 p}, p}}+|\lambda|^{\frac{1}{2}-\frac{1}{2 p}}\left\|f_{k}\right\|_{H}\right) .
\end{aligned}
$$

Proof. Condition 1) implies that, for $|\arg \lambda| \leq \varphi<\pi\left\|(A+\lambda I)^{-1}\right\| \leq C(1+|\lambda|)^{-1}$. Then, by [13, lemma 5.4.2/6], for $|\arg \lambda| \leq \varphi<\pi$ there exists an analytic, for $x>0$, and a strongly continuous, for $x \geq 0$, semigroup $e^{-x(A+\lambda I)^{1 / 2}}$. By [13, lemma 5.4.2/1], for the function $u(x)$ to be a solution of the equation (2.1), belonging to $W_{p}^{2}((0,1) ; H(A), H), p \in(1, \infty)$, it is necessary and sufficient that, for $|\arg \lambda| \leq \varphi<\pi$,

$$
u(x)=e^{-x(A+\lambda I)^{1 / 2}} g_{1}+e^{-(1-x)(A+\lambda I)^{1 / 2}} g_{2},
$$


where $g_{1}, g_{2} \in(H(A), H)_{\frac{1}{2 p}, p}$.

Require that the function of the form (2.4) satisfies the boundary conditions in (2.2). Then for the elements $g_{1}$ and $g_{2}$ we get the following system:

$$
\begin{gathered}
{\left[\lambda I+\alpha(A+\lambda I)^{1 / 2}+B_{1} e^{-(A+\lambda I)^{1 / 2}}\right] g_{1}+} \\
+\left[\left(\lambda I-\alpha(A+\lambda I)^{1 / 2}\right) e^{-(A+\lambda I)^{1 / 2}}+B_{1}\right] g_{2}=f_{1}, \\
{\left[\left(\lambda I-\beta(A+\lambda I)^{1 / 2}\right) e^{-(A+\lambda I)^{1 / 2}}+B_{2}\right] g_{1}+} \\
{\left[\lambda I+\beta(A+\lambda I)^{1 / 2}+B_{2} e^{-(A+\lambda I)^{1 / 2}}\right] g_{2}=f_{2} .}
\end{gathered}
$$

We write the system $(2.5)$ in the space $\mathbb{H}:=(H(A), H)_{\frac{1}{2}+\frac{1}{2 p}, p} \dot{+}(H(A), H)_{\frac{1}{2}+\frac{1}{2 p}, p}$ in the following operator equation form

$$
(A(\lambda)+R(\lambda))\left(\begin{array}{l}
g_{1} \\
g_{2}
\end{array}\right)=\left(\begin{array}{l}
f_{1} \\
f_{2}
\end{array}\right) .
$$

Here $A(\lambda)$ and $R(\lambda)$ are operator-matrices of dimension $2 \times 2$ :

$$
\begin{gathered}
A(\lambda):=\left(\begin{array}{cc}
\lambda I+\alpha(A+\lambda I)^{1 / 2} & 0 \\
B_{2} & \lambda I+\beta(A+\lambda I)^{1 / 2}
\end{array}\right), \\
D(A(\lambda)):=(H(A), H)_{\frac{1}{2 p}, p} \dot{+}(H(A), H)_{\frac{1}{2 p}, p}:=\mathbb{H}_{1}
\end{gathered}
$$

and

$$
\begin{gathered}
R(\lambda):= \\
=\left(\begin{array}{cc}
B_{1} e^{-(A+\lambda I)^{1 / 2}} & \left(\lambda I-\alpha(A+\lambda I)^{1 / 2}\right) e^{-(A+\lambda I)^{1 / 2}}+B_{1} \\
\left(\lambda I-\beta(A+\lambda I)^{1 / 2}\right) e^{-(A+\lambda I)^{1 / 2}} & B_{2} e^{-(A+\lambda I)^{1 / 2}}
\end{array}\right), \\
D(R(\lambda)):=\mathbb{H} .
\end{gathered}
$$

Show that the operator $A(\lambda)$ in the space $\mathbb{H}$ for $\lambda$ from the angle $|\arg \lambda| \leq \varphi-\varepsilon$, where $\varepsilon>0$ is a sufficiently small number, has a bounded inverse $A(\lambda)^{-1}$ acting from $\mathbb{H}$ into $\mathbb{H}_{1}$ and that the estimate

$$
\left\|A(\lambda)^{-1}\right\|_{B\left(\mathbb{H}, \mathbb{H}_{1}\right)} \leq C(\varphi, \varepsilon),
$$

where $C(\varphi, \varepsilon)>0$ is a constant independent of $\lambda$, is satisfied. Since the inverse operator $A(\lambda)^{-1}$ is formally given of the form

$$
\begin{gathered}
A(\lambda)^{-1}= \\
=\left(\begin{array}{cc}
{\left[\lambda I+\alpha(A+\lambda I)^{1 / 2}\right]^{-1}} & 0 \\
-\left[\lambda I+\beta(A+\lambda I)^{1 / 2}\right]^{-1} B_{2}\left[\lambda I+\alpha(A+\lambda I)^{1 / 2}\right]^{-1} & {\left[\lambda I+\beta(A+\lambda I)^{1 / 2}\right]^{-1}}
\end{array}\right)
\end{gathered}
$$

then for that it suffices to show that 
a) the operator $\left[\lambda I+\alpha(A+\lambda I)^{1 / 2}\right]^{-1}$ for $\lambda$ from the angle $|\arg \lambda| \leq \varphi-\varepsilon$, where $\varepsilon>0$ is a sufficiently small number, is bounded from $(H(A), H)_{\frac{1}{2}+\frac{1}{2 p}, p}$ into $(H(A), H)_{\frac{1}{2 p}, p}$ and that the estimate is true

$$
\left\|\left[\lambda I+\alpha(A+\lambda I)^{1 / 2}\right]^{-1}\right\|_{B\left((H(A), H)_{\frac{1}{2}+\frac{1}{2 p}, p},(H(A), H)_{\frac{1}{2 p}, p}\right)} \leq C(\varphi, \varepsilon) ;
$$

b) the operator $\left[\lambda I+\beta(A+\lambda I)^{1 / 2}\right]^{-1} B_{2}\left[\lambda I+\alpha(A+\lambda I)^{1 / 2}\right]^{-1}$ for $\lambda$ from the angle $|\arg \lambda| \leq \varphi-\varepsilon$, where $\varepsilon>0$ is a sufficiently small number is bounded from $(H(A), H)_{\frac{1}{2}+\frac{1}{2 p}, p}$ into $(H(A), H)_{\frac{1}{2 p}, p}$ and that the estimate is true

$$
\begin{gathered}
\|\left[\lambda I+\beta(A+\lambda I)^{1 / 2}\right]^{-1} \times \\
\times B_{2}\left[\lambda I+\alpha(A+\lambda I)^{1 / 2}\right]^{-1} \|_{B\left((H(A), H)_{\frac{1}{2}+\frac{1}{2 p}, p}(H(A), H)_{\frac{1}{2 p}, p}\right)} \leq C(\varphi, \varepsilon) .
\end{gathered}
$$

Prove statement a). In [6], it was shown that for $\lambda$ from the angle $|\arg \lambda| \leq \varphi$, the operator $(A+\lambda I)^{-1 / 2}$ is bounded from $(H(A), H)_{\frac{1}{2}+\frac{1}{2 p}, p}$ into $(H(A), H)_{\frac{1}{2 p}, p}$ and the estimate is satisfied

$$
\left\|(A+\lambda I)^{-1 / 2}\right\|_{B\left((H(A), H)_{\frac{1}{2}+\frac{1}{2 p}, p},(H(A), H)_{\frac{1}{2 p}, p}\right.} \leq C,
$$

where $C>0$ is a constant independent of $\lambda$. In paper [14], it was proved that for $\lambda$ from the angle $|\arg \lambda| \leq \varphi-\varepsilon$, where $\varepsilon>0$ is a sufficiently small number, the operator $\left[\alpha I+\lambda(A+\lambda I)^{-1 / 2}\right]^{-1}$, subject to conditions 1) and 2), is bounded from $H$ into $H$ and the estimate is satisfied

$$
\left\|\left[\alpha I+\lambda(A+\lambda I)^{-1 / 2}\right]^{-1}\right\|_{B(H)} \leq C(\varphi, \varepsilon) .
$$

Then, by (2.11),

$$
\begin{gathered}
\left\|\left[\alpha I+\lambda(A+\lambda I)^{-1 / 2}\right]^{-1}\right\|_{B(H(A))}=\left\|A\left[\alpha I+\lambda(A+\lambda I)^{-1 / 2}\right]^{-1} A^{-1}\right\|_{B(H)}= \\
=\left\|\left[\alpha I+\lambda(A+\lambda I)^{-1 / 2}\right]^{-1}\right\|_{B(H)} \leq C(\varphi, \varepsilon) .
\end{gathered}
$$

By virtue of the interpolation theorem 1.1, from the estimates (2.11), (2.12), it follows that the operator $\left[\alpha I+\lambda(A+\lambda I)^{-1 / 2}\right]^{-1}$ for $\lambda$ from the angle $|\arg \lambda| \leq$ $\leq \varphi-\varepsilon$, where $\varepsilon>0$ is a sufficiently small number, is bounded from $(H(A), H)_{\theta, p}$ into $(H(A), H)_{\theta, p}$, for any $\theta \in(0,1)$, and the estimate is satisfied

$$
\left\|\left[\alpha I+\lambda(A+\lambda I)^{-1 / 2}\right]^{-1}\right\|_{B\left((H(A), H)_{\theta, p}\right)} \leq C(\varphi, \varepsilon) .
$$

In particular, for $\theta=\frac{1}{2 p}$, we have

$$
\left\|\left[\alpha I+\lambda(A+\lambda I)^{-1 / 2}\right]^{-1}\right\|_{B\left((H(A), H)_{\frac{1}{2 p}, p}\right)} \leq C(\varphi, \varepsilon) .
$$


From the representation

$$
\left[\lambda I+\alpha(A+\lambda I)^{1 / 2}\right]^{-1}=\left[\alpha I+\lambda(A+\lambda I)^{-1 / 2}\right]^{-1}(A+\lambda I)^{-1 / 2},
$$

by virtue of the estimates $(2.10),(2.14)$, it follows that for $\lambda$ from the angle $|\arg \lambda| \leq \varphi-\varepsilon$, where $\varepsilon>0$ is a sufficiently small number, we have the estimate (2.8). This proves the statement a).

Prove the statement b). It was shown in [6] that, the operator $B_{2}$, subject to the condition 4), is bounded from $(H(A), H)_{\frac{1}{2 p}, p}$ into $(H(A), H)_{\frac{1}{2}+\frac{1}{2 p}, p}$ and the estimate is satisfied

$$
\left\|B_{2}\right\|_{B\left((H(A), H)_{\frac{1}{2 p}, p},(H(A), H)_{\frac{1}{2}+\frac{1}{2 p}, p}\right)} \leq C .
$$

Then, the validity of the statement b) follows from the estimates (2.8) and (2.16). The estimate (2.7) follows from the estimates (2.8), (2.9).

Then, from equation (2.6), we have

$$
\left(I+A(\lambda)^{-1} R(\lambda)\right)\left(\begin{array}{l}
g_{1} \\
g_{2}
\end{array}\right)=A(\lambda)^{-1}\left(\begin{array}{l}
f_{1} \\
f_{2}
\end{array}\right) .
$$

From the representations of $A(\lambda)^{-1}$ and $R(\lambda)$, we can see that the product $A(\lambda)^{-1} R(\lambda)$ is an operator-matrix of dimension $2 \times 2$ :

$$
A(\lambda)^{-1} R(\lambda)=\left(\begin{array}{ll}
K_{11}(\lambda) & K_{12}(\lambda) \\
K_{21}(\lambda) & K_{22}(\lambda)
\end{array}\right),
$$

where

$$
\begin{gathered}
K_{11}(\lambda):=\left[\lambda I+\alpha(A+\lambda I)^{1 / 2}\right]^{-1} B_{1} e^{-(A+\lambda I)^{1 / 2}}, \\
K_{12}(\lambda):=\left[\lambda I+\alpha(A+\lambda I)^{1 / 2}\right]^{-1}\left[B_{1}+\left(\lambda I-\alpha(A+\lambda I)^{1 / 2}\right) e^{-(A+\lambda I)^{1 / 2}}\right], \\
\left.K_{21}(\lambda):=-\left[\lambda I+\beta(A+\lambda I)^{1 / 2}\right]^{-1} B_{2}\left[\lambda I+\alpha(A+\lambda I)^{1 / 2}\right)\right]^{-1} B_{1} e^{-(A+\lambda I)^{1 / 2}}+ \\
+\left[\lambda I+\beta(A+\lambda I)^{1 / 2}\right]^{-1}\left(\lambda I-\beta(A+\lambda I)^{1 / 2}\right) e^{-(A+\lambda I)^{1 / 2}}, \\
\left.K_{22}(\lambda):=-\left[\lambda I+\beta(A+\lambda I)^{1 / 2}\right]^{-1} B_{2}\left[\lambda I+\alpha(A+\lambda I)^{1 / 2}\right)\right]^{-1} \times \\
\times\left\{B_{1}+\left(\lambda I-\alpha(A+\lambda I)^{1 / 2}\right) e^{-(A+\lambda I)^{1 / 2}}\right\}+\left[\lambda I+\beta(A+\lambda I)^{1 / 2}\right]^{-1} B_{2} e^{-(A+\lambda I)^{1 / 2}} .
\end{gathered}
$$

Show that for sufficiently large $\lambda$ from the angle $|\arg \lambda| \leq \varphi-\varepsilon$, where $\varepsilon>0$ is a sufficiently small number, the operator $A(\lambda)^{-1} R(\lambda)$ is bounded from $\mathbb{H}_{1}$ into $\mathbb{H}_{1}$ and that the estimate is true

$$
\left\|A(\lambda)^{-1} R(\lambda)\right\|_{B\left(\mathbb{H}_{1}\right)} \leq \frac{C(\varphi, \varepsilon)}{(1+|\lambda|)^{1 / 2}}<1 .
$$

In order to prove the estimate (2.18), it suffices to show that all operators which appear in the operator-matrix $A(\lambda)^{-1} R(\lambda)$ for sufficiently large $\lambda$ from the angle $|\arg \lambda| \leq \varphi-\varepsilon$, where $\varepsilon>0$ is a sufficiently small number, are bounded 
from $(H(A), H)_{\frac{1}{2 p}, p}$ into $(H(A), H)_{\frac{1}{2 p}, p}$. Show this, for example, for the operator $K_{22}(\lambda)$.

According to theorem 1.1, it suffices to show that the operator $K_{22}(\lambda)$ for sufficiently large $\lambda$ from the angle $|\arg \lambda| \leq \varphi-\varepsilon$, where $\varepsilon>0$ is a sufficiently small number, is bounded from $H$ into $H$ and from $H(A)$ into $H(A)$. More precisely, we have to estimate the norms $\left\|K_{22}(\lambda)\right\|_{B(H)}$ and $\left\|K_{22}(\lambda)\right\|_{B(H(A))}$ for the mentioned $\lambda$. Before estimating these norms, using the representation (2.15), rewrite the operators $K_{22}(\lambda)$ and $A K_{22}(\lambda) A^{-1}$ (since $\left\|K_{22}(\lambda)\right\|_{B(H(A))}=\left\|A K_{22}(\lambda) A^{-1}\right\|_{B(H)}$ ) in the form

$$
\begin{gathered}
K_{22}(\lambda)=-\left[\beta I+\lambda(A+\lambda I)^{-1 / 2}\right]^{-1}(A+\lambda I)^{-1 / 2} B_{2} A^{-1 / 2} A^{1 / 2}(A+\lambda I)^{-1 / 2} \times \\
\times\left[\alpha I+\lambda(A+\lambda I)^{-1 / 2}\right]^{-1}\left[B_{1}+\left(\lambda(A+\lambda I)^{-1 / 2}-\alpha I\right)(A+\lambda I)^{1 / 2} e^{-(A+\lambda I)^{1 / 2}}\right]+ \\
+\left[\beta I+\lambda(A+\lambda I)^{-1 / 2}\right]^{-1}(A+\lambda I)^{-1 / 2} B_{2} A^{-1 / 2} A^{1 / 2} e^{-(A+\lambda I)^{1 / 2}}, \\
A K_{22}(\lambda) A^{-1}= \\
=-\left[\beta I+\lambda(A+\lambda I)^{-1 / 2}\right]^{-1} A^{1 / 2}(A+\lambda I)^{-1 / 2} A^{1 / 2} B_{2} A^{-1}(A+\lambda I)^{-1 / 2} \times \\
\times\left[\alpha I+\lambda(A+\lambda I)^{-1 / 2}\right]^{-1}\left[A B_{1} A^{-1}+\right. \\
\left.+\left(\lambda(A+\lambda I)^{-1 / 2}-\alpha I\right)(A+\lambda I)^{1 / 2} e^{-(A+\lambda I)^{1 / 2}}\right]+ \\
+\left[\beta I+\lambda(A+\lambda I)^{-1 / 2}\right]^{-1} A^{1 / 2}(A+\lambda I)^{-1 / 2} A^{1 / 2} B_{2} A^{-1} e^{-(A+\lambda I)^{1 / 2}} .
\end{gathered}
$$

By virtue of [13, lemma 5.4.2/6], the estimate (2.11), conditions 3 ) and 4), for sufficiently large $\lambda$ from the angle $|\arg \lambda| \leq \varphi-\varepsilon$, where $\varepsilon>0$ is a sufficiently small number, we have

$$
\begin{gathered}
\left\|K_{22}(\lambda)\right\|_{B(H)} \leq \\
\leq\left\|\left[\beta I+\lambda(A+\lambda I)^{-1 / 2}\right]^{-1}\right\|_{B(H)}\left\|(A+\lambda I)^{-1 / 2}\right\|_{B(H)}\left\|B_{2} A^{-1 / 2}\right\|_{B(H)} \times \\
\times\left\|A^{1 / 2}(A+\lambda I)^{-1 / 2}\right\|_{B(H)}\left\|\left[\alpha I+\lambda(A+\lambda I)^{-1 / 2}\right]^{-1}\right\|_{B(H)} \times \\
\times\left[\left\|B_{1}\right\|_{B(H)}+\left\|\lambda(A+\lambda I)^{-1 / 2}-\alpha I\right\|_{B(H)} \times\right. \\
\left.\times\left\|(A+\lambda I)^{1 / 2} e^{-(A+\lambda I)^{1 / 2}}\right\|_{B(H)}\right]+\left\|\left[\beta I+\lambda(A+\lambda I)^{-1 / 2}\right]^{-1}\right\|_{B(H)} \times \\
\times\left\|(A+\lambda I)^{-1 / 2}\right\|_{B(H)}\left\|B_{2} A^{-1 / 2}\right\|_{B(H)}\left\|A^{1 / 2} e^{-(A+\lambda I)^{1 / 2}}\right\|_{B(H)}{\frac{C(\varphi, \varepsilon)}{|\lambda|^{1 / 2}}}^{(2.19)} \\
\left\|K_{22}(\lambda)\right\|_{B(H(A))}=\left\|A K_{22}(\lambda) A^{-1}\right\|_{B(H)} \leq\left\|\left[\beta I+\lambda(A+\lambda I)^{-1 / 2}\right]^{-1}\right\|_{B(H)} \times \\
\times\left\|A^{1 / 2}(A+\lambda I)^{-1 / 2}\right\|_{B(H)}\left\|A^{1 / 2} B_{2} A^{-1}\right\|_{B(H)}\left\|(A+\lambda I)^{-1 / 2}\right\|_{B(H)} \times \\
\times\left\|\left[\alpha I+\lambda(A+\lambda I)^{-1 / 2}\right]^{-1}\right\|_{B(H)}\left[\left\|A B_{1} A^{-1}\right\|_{B(H)}+\right. \\
\left.+\left\|\left(\lambda(A+\lambda I)^{-1 / 2}-\alpha I\right)\right\|_{B(H)}\left\|(A+\lambda I)^{1 / 2} e^{-(A+\lambda I)^{1 / 2}}\right\|_{B(H)}\right]+ \\
\left.+\lambda(A+\lambda I)^{-1 / 2}\right]^{-1}\left\|_{B(H)}\right\| A^{1 / 2}(A+\lambda I)^{-1 / 2}\left\|_{B(H)}\right\| A^{1 / 2} B_{2} A^{-1} \|_{B(H)} \times
\end{gathered}
$$




$$
\times\left\|e^{-(A+\lambda I)^{1 / 2}}\right\|_{B(H)} \leq \frac{C(\varphi, \varepsilon)}{|\lambda|^{1 / 2}} .
$$

By theorem 1.1, from the estimates (2.19) and (2.20), it follows that the operator $K_{22}(\lambda)$ for sufficiently large $\lambda$ from the angle $|\arg \lambda| \leq \varphi-\varepsilon$, is bounded from $(H(A), H)_{\theta, p}$ into $(H(A), H)_{\theta, p}$ for any $\theta \in(0,1)$ and, in particular, from $(H(A), H)_{\frac{1}{2 p}, p}$ into $(H(A), H)_{\frac{1}{2 p}, p}$ (for $\left.\theta=\frac{1}{2 p}\right)$ and the estimate is true

$$
\left\|K_{22}(\lambda)\right\|_{B\left((H(A) H)_{\frac{1}{2 p}, p}\right)} \leq \frac{C(\varphi, \varepsilon)}{(1+|\lambda|)^{1 / 2}} .
$$

In the same way, we show that the remaning operators, participating in the operator-matrix $A(\lambda)^{-1} R(\lambda)$, for sufficiently large $\lambda$ from the angle $|\arg \lambda| \leq \varphi-\varepsilon$ are bounded from $(H(A), H)_{\frac{1}{2 p}, p}$ into $(H(A), H)_{\frac{1}{2 p}, p}$, and the estimate $(2.21)$ is satisfied. Thus, the estimate (2.18) holds.

Hence, by the Neumann identity, for sufficiently large $\lambda$ from the angle $|\arg \lambda| \leq$ $\varphi-\varepsilon$

$$
\left(I+A(\lambda)^{-1} R(\lambda)\right)^{-1}=I+\sum_{k=1}^{\infty}(-1)^{k}\left(A(\lambda)^{-1} R(\lambda)\right)^{k},
$$

where the series in the right-hand side of (2.22) converges in the norm of the space of bounded operators acting in $\mathbb{H}_{1}$.

By (2.18), for sufficiently large $\lambda$ from the angle $|\arg \lambda| \leq \varphi-\varepsilon$, from (2.17) we have

$$
\left(\begin{array}{l}
g_{1} \\
g_{2}
\end{array}\right)=\left(I+A(\lambda)^{-1} R(\lambda)\right)^{-1} A(\lambda)^{-1}\left(\begin{array}{c}
f_{1} \\
f_{2}
\end{array}\right) .
$$

Hence, for sufficiently large $\lambda$ from the angle $|\arg \lambda| \leq \varphi-\varepsilon$, the elements $g_{1}$ and $g_{2}$ can be represented in the form

$$
g_{k}=\left(C_{k 1}(\lambda)+R_{k 1}(\lambda)\right) f_{1}+\left(C_{k 2}(\lambda)+R_{k 2}(\lambda)\right) f_{2}, \quad k=1,2,
$$

where

$$
\begin{gathered}
C_{11}(\lambda)=\left[\lambda I+\alpha(A+\lambda I)^{1 / 2}\right]^{-1}, C_{12}(\lambda)=0, \\
C_{21}(\lambda)=-\left[\lambda I+\beta(A+\lambda I)^{1 / 2}\right]^{-1} B_{2}\left[\lambda I+\alpha(A+\lambda I)^{1 / 2}\right]^{-1}, \\
C_{22}(\lambda)=\left[\lambda I+\beta(A+\lambda I)^{1 / 2}\right]^{-1},
\end{gathered}
$$

$R_{k j}(\lambda)(k, j=1,2)$ are some bounded operators acting from $(H(A), H)_{\frac{1}{2}+\frac{1}{2 p}, p}$ into $(H(A), H)_{\frac{1}{2 p}, p}$, for $|\arg \lambda| \leq \varphi-\varepsilon,|\lambda| \rightarrow \infty$. Furthermore, from the estimates (2.7) and (2.18), it follows that for $|\arg \lambda| \leq \varphi-\varepsilon,|\lambda| \rightarrow \infty$,

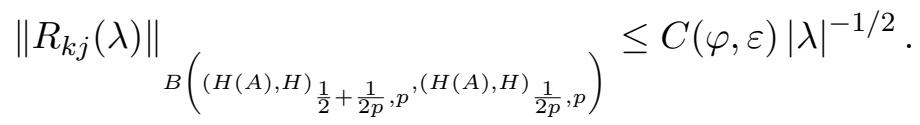

From the representations of $A(\lambda)^{-1}$ and $A(\lambda)^{-1} R(\lambda)$ it also follows that for sufficiently large $\lambda$ from the angle $|\arg \lambda| \leq \varphi-\varepsilon$, for the operator $R_{k j}(\lambda)$ we have the estimate

$$
\left\|R_{k j}(\lambda)\right\|_{B(H)} \leq C(\varphi, \varepsilon)|\lambda|^{-1 / 2} \text {. }
$$


Substituting (2.23) into (2.4), we get

$$
\begin{gathered}
u(x)=\sum_{k=1}^{2}\left\{e^{-x(A+\lambda I)^{1 / 2}}\left(C_{1 k}(\lambda)+R_{1 k}(\lambda)\right)+\right. \\
\left.+e^{-(1-x)(A+\lambda I)^{1 / 2}}\left(C_{2 k}(\lambda)+R_{2 k}(\lambda)\right)\right\} f_{k} .
\end{gathered}
$$

Note that for sufficiently large $\lambda$ from the angle $|\arg \lambda| \leq \varphi-\varepsilon$, from (2.26) we have

$$
\begin{aligned}
& |\lambda|\|u\|_{L_{p}((0,1) ; H)}+\left\|u^{\prime \prime}\right\|_{L_{p}((0,1) ; H)}+\|A u\|_{L_{p}((0,1) ; H)} \leq \\
& \leq C(\varphi, \varepsilon) \sum_{k=1}^{2}\left\{| \lambda | \left[\left(\int_{0}^{1}\left\|e^{-x(A+\lambda I)^{1 / 2}} C_{1 k}(\lambda) f_{k}\right\|_{H}^{p} d x\right)^{1 / p}+\right.\right. \\
& +\left(\int_{0}^{1}\left\|e^{-x(A+\lambda I)^{1 / 2}} R_{1 k}(\lambda) f_{k}\right\|_{H}^{p} d x\right)^{1 / p}+\left(\int_{0}^{1}\left\|e^{-(1-x)(A+\lambda I)^{1 / 2}} C_{2 k}(\lambda) f_{k}\right\|_{H}^{p} d x\right)^{1 / p} \\
& \left.+\left(\int_{0}^{1}\left\|e^{-(1-x)(A+\lambda I)^{1 / 2}} R_{2 k}(\lambda) f_{k}\right\|_{H}^{p} d x\right)^{1 / p}\right]+\left(1+\left\|A(A+\lambda I)^{-1}\right\|\right) \times \\
& \times\left[\left(\int_{0}^{1}\left\|(A+\lambda I) e^{-x(A+\lambda I)^{1 / 2}} C_{1 k}(\lambda) f_{k}\right\|_{H}^{p} d x\right)^{1 / p}+\right. \\
& +\left(\int_{0}^{1}\left\|(A+\lambda I) e^{-x(A+\lambda I)^{1 / 2}} R_{1 k}(\lambda) f_{k}\right\|_{H}^{p} d x\right)^{1 / p}+ \\
& +\left(\int_{0}^{1}\left\|(A+\lambda I) e^{-(1-x)(A+\lambda I)^{1 / 2}} C_{2 k}(\lambda) f_{k}\right\|_{H}^{p} d x\right)^{1 / p}+ \\
& \left.\left.+\left(\int_{0}^{1}\left\|(A+\lambda I) e^{-(1-x)(A+\lambda I)^{1 / 2}} R_{2 k}(\lambda) f_{k}\right\|_{H}^{p} d x\right)^{1 / p}\right]\right\} .
\end{aligned}
$$

By virite of [13, theorem 5.4.2/1], the representation (2.15), the estimate (2.11), and the estimate (2.13), for $\theta=\frac{1}{2}+\frac{1}{2 p}$, for sufficiently large $\lambda$ from the angle $|\arg \lambda| \leq \varphi-\varepsilon$, for the first summand of the right-hand side of the inequality (2.27) we have

$$
|\lambda| \sum_{k=1}^{2}\left(\int_{0}^{1}\left\|e^{-x(A+\lambda I)^{1 / 2}} C_{1 k}(\lambda) f_{k}\right\|_{H}^{p} d x\right)^{1 / p}=
$$




$$
\begin{aligned}
& =|\lambda|\left(\int_{0}^{1}\left\|e^{-x(A+\lambda I)^{1 / 2}}\left[\lambda I+\alpha(A+\lambda I)^{1 / 2}\right]^{-1} f_{1}\right\|_{H}^{p} d x\right)^{1 / p} \leq \\
& \leq|\lambda|\left\|(A+\lambda I)^{-1}\right\|_{B(H)} \times \\
& \times\left(\int_{0}^{1}\left\|(A+\lambda I) e^{-x(A+\lambda I)^{1 / 2}}(A+\lambda I)^{-1 / 2}\left[\alpha I+\lambda(A+\lambda I)^{-1 / 2}\right]^{-1} f_{1}\right\|_{H}^{p} d x\right)^{1 / p} \\
& \leq C\left(\int_{0}^{1}\left\|(A+\lambda I)^{1 / 2} e^{-x(A+\lambda I)^{1 / 2}}\left[\alpha I+\lambda(A+\lambda I)^{-1 / 2}\right]^{-1} f_{1}\right\|_{H}^{p} d x\right)^{1 / p} \leq \\
& \leq C\left(\left\|\left[\alpha I+\lambda(A+\lambda I)^{-1 / 2}\right]^{-1} f_{1}\right\|_{(H(A), H)_{\frac{1}{2}+\frac{1}{2 p}, p}}+\right. \\
& \left.+|\lambda|^{\frac{1}{2}-\frac{1}{2 p}}\left\|\left[\alpha I+\lambda(A+\lambda I)^{-1 / 2}\right]^{-1} f_{1}\right\|_{H}\right) \leq \\
& \leq C(\varphi, \varepsilon)\left(\left\|f_{1}\right\|_{(H(A), H)_{\frac{1}{2}+\frac{1}{2 p}, p}}+|\lambda|^{\frac{1}{2}-\frac{1}{2 p}}\left\|f_{1}\right\|_{H}\right) .
\end{aligned}
$$

By virtue of $[13$, theorem 5.4.2/1] and estimates (2.24), (2.25), for sufficiently large $\lambda$ from the angle $|\arg \lambda| \leq \varphi-\varepsilon$, for the second summand of the right-hand part of the inequality (2.27) we have

$$
\begin{gathered}
|\lambda| \sum_{k=1}^{2}\left(\int_{0}^{1}\left\|e^{-x(A+\lambda I)^{1 / 2}} R_{1 k}(\lambda) f_{k}\right\|_{H}^{p} d x\right)^{1 / p} \leq \\
\leq|\lambda|\left\|(A+\lambda I)^{-1}\right\|_{B(H)} \sum_{k=1}^{2}\left(\int_{0}^{1}\left\|(A+\lambda I) e^{-x(A+\lambda I)^{1 / 2}} R_{1 k}(\lambda) f_{k}\right\|_{H}^{p} d x\right)^{1 / p} \leq \\
\leq C \sum_{k=1}^{2}\left(\|\left(R_{1 k}(\lambda) f_{k}\left\|_{(H(A), H)_{\frac{1}{2 p}, p}}+|\lambda|^{1-\frac{1}{2 p}}\right\| R_{1 k}(\lambda) f_{k} \|_{H}\right) \leq\right. \\
\leq C(\varphi, \varepsilon) \sum_{k=1}^{2}\left(\left\|f_{k}\right\|_{(H(A), H)_{\frac{1}{2}+\frac{1}{2 p}, p}}+|\lambda|^{\frac{1}{2}-\frac{1}{2 p}}\left\|f_{k}\right\|_{H}\right) .
\end{gathered}
$$

In the same way, we can estimate remaining summands of the right-hand side of the inequality (2.27) and get the required estimate (2.3). Theorem 2.1 is proved. 


\section{Nonhomogeneous equation}

Now, in a separable Hilbert space $H$, we consider a boundary value problem for the nonhomogeneous equation with a parameter, i.e., the problem

$$
\begin{gathered}
L(\lambda) u:=\lambda u(x)-u^{\prime \prime}(x)+A u(x)=f(x), \quad x \in(0,1), \\
L_{1}(\lambda) u:=\lambda u(0)-\alpha u^{\prime}(0)+B_{1} u(1)=f_{1}, \\
L_{2}(\lambda) u:=\lambda u(1)+\beta u^{\prime}(1)+B_{2} u(0)=f_{2} .
\end{gathered}
$$

Theorem 3.1. Let the all conditions of theorem 2.1 be fulfilled.

Then, the operator $\mathbb{L}(\lambda): u \rightarrow \mathbb{L}(\lambda) u:=\left(L(\lambda) u, L_{1}(\lambda) u, L_{2}(\lambda) u\right)$ for sufficiently large $\lambda$ from the angle $|\arg \lambda| \leq \varphi-\varepsilon$, where $\varepsilon>0$ is a sufficiently small number, is an isomorphism from $\bar{W}_{p}^{2}((0,1) ; H(A), H)$ into $L_{p}((0,1) ; H) \dot{+}$ $(H(A), H)_{\frac{1}{2}+\frac{1}{2 p}, p} \dot{+}(H(A), H)_{\frac{1}{2}+\frac{1}{2 p}, p}$ and, for these $\lambda$, the following estimate for the solution of the problem (3.1), (3.2) is valid:

$$
\begin{gathered}
|\lambda|\|u\|_{L_{p}((0,1) ; H)}+\left\|u^{\prime \prime}\right\|_{L_{p}((0,1) ; H)}+\|A u\|_{L_{p}((0,1) ; H)} \leq \\
\leq C(\varphi, \varepsilon)\left[|\lambda|\|f\|_{L_{p}((0,1) ; H)}+\sum_{k=1}^{2}\left(\left\|f_{k}\right\|_{(H(A), H)_{\frac{1}{2}+\frac{1}{2 p}, p}}+|\lambda|^{\frac{1}{2}-\frac{1}{2 p}}\left\|f_{k}\right\|_{H}\right)\right] .
\end{gathered}
$$

Proof. Injectivity of the mapping $\mathbb{L}(\lambda)$ follows from theorem 2.1 , since a homogeneous boundary value problem, corresponding to the boundary value problem (3.1), (3.2) for sufficiently large $\lambda$ from the angle $|\arg \lambda| \leq \varphi-\varepsilon$, has only a trivial solution. Thus, it suffices to show that $\mathbb{L}(\lambda)$ is surjective, i.e., for any $f(x) \in L_{p}((0,1) ; H)$ and any $f_{1}, f_{2} \in(H(A), H)_{\frac{1}{2}+\frac{1}{2 p}, p}$, there exists a solution of the problem (3.1), (3.2), belonging to $W_{p}^{2}((0,1) ; H(A), H)$. Determine $\widetilde{f}(x)=f(x)$ if $x \in[0,1]$ and $\widetilde{f}(x)=0$ if $x \notin[0,1]$ The solution of the problem $(3.1),(3.2)$ is represented as the sum $u(x)=u_{1}(x)+u_{2}(x)$, where $u_{1}(x)$ is a contraction on $[0,1]$ of the solution $\widetilde{u}_{1}(x)$ of the equation

$$
L(\lambda) \widetilde{u}_{1}(x)=\widetilde{f}(x), \quad x \in \mathbb{R}=(-\infty,+\infty),
$$

while $u_{2}(x)$ is a solution of the problem

$$
L(\lambda) u_{2}=0, \quad L_{k}(\lambda) u_{2}=f_{k}-L_{k}(\lambda) u_{1}, \quad k=1,2 .
$$

As it was shown in the proof of theorem 5.4.4 from [13], the solution of the equation (3.4) is given by the following formula

$$
\widetilde{u}_{1}(x)=\frac{1}{2 \pi} \int_{\mathbb{R}} e^{i \mu x} L(\lambda, i \mu)^{-1} F \tilde{f}(\mu) d \mu .
$$

Here $F \widetilde{f}$ is the Fourier transform of the function $\widetilde{f}(x)$, and $L(\lambda, \sigma)$ is a characteristic operator pencil of the equation (3.4), i.e., $L(\lambda, \sigma)=-\sigma^{2} I+A+\lambda I$. It was proved [13, theorem 5.4.4] that there exists a solution of the equation (3.4), belonging to $W_{p}^{2}((0,1) ; H(A), H)$, and for this solution the estimate is valid

$$
|\lambda|\left\|\widetilde{u}_{1}\right\|_{L_{p}(\mathbb{R} ; H)}+\left\|\widetilde{u}_{1}\right\|_{W_{p}^{2}(\mathbb{R} ; H(A), H)} \leq\|\widetilde{f}\|_{L_{p}(\mathbb{R} ; H)},|\arg \lambda| \leq \varphi<\pi .
$$


Therefore, $u_{1} \in W_{p}^{2}((0,1) ; H(A), H)$. From $(3.6)$, for $|\arg \lambda| \leq \varphi$, we have

$$
|\lambda|\left\|u_{1}\right\|_{L_{p}((0,1) ; H)}+\left\|u_{1}\right\|_{W_{p}^{2}((0,1) ; H(A), H)} \leq C\|f\|_{L_{p}((0,1) ; H)} \cdot
$$

By $[12$, theorem 1.8.2] (see also [13, theorem 1.7.7/1 ]) and the inequality (3.7), we have

$$
u_{1}^{(s)}\left(x_{0}\right) \in(H(A), H)_{\frac{s}{2}+\frac{1}{2 p}, p}, \quad \forall x_{0} \in[0,1], s=0,1 .
$$

From conditions 3) and 4) of theorem 2.1 (see also theorem 1.1 and (2.16)), it follows that $L_{k}(\lambda) u_{1} \in(H(A), H)_{\frac{1}{2}+\frac{1}{2 p}, p}$, since $(H(A), H)_{\frac{1}{2 p}, p} \subset(H(A), H)_{\frac{1}{2}+\frac{1}{2 p}, p}$.

Thus, by virtue of theorem 2.1, for sufficiently large $\lambda$ from the angle $|\arg \lambda| \leq$ $\varphi-\varepsilon$, the problem (3.5) has a unique solution $u_{2}(x)$ that belongs to $W_{p}^{2}((0,1) ; H(A), H)$. Furthermore, for the solution of the problem (3.5) for $|\arg \lambda| \leq \varphi-\varepsilon,|\lambda| \rightarrow \infty$, we have

$$
\begin{aligned}
& |\lambda|\left\|u_{2}\right\|_{L_{p}((0,1) ; H)}+\left\|u_{2}^{\prime \prime}\right\|_{L_{p}((0,1) ; H)}+\left\|A u_{2}\right\|_{L_{p}((0,1) ; H)} \leq \\
& \leq C(\varphi, \varepsilon) \sum_{k=1}^{2}\left(\left\|f_{k}-L_{k}(\lambda) u_{1}\right\|_{(H(A), H)_{\frac{1}{2}+\frac{1}{2 p}, p}}+|\lambda|^{\frac{1}{2}-\frac{1}{2 p}}\left\|f_{k}-L_{k}(\lambda) u_{1}\right\|_{H}\right) \leq \\
& \leq C(\varphi, \varepsilon) \sum_{k=1}^{2}\left(\left\|f_{k}\right\|_{(H(A), H)_{\frac{1}{2}+\frac{1}{2 p}, p}}+|\lambda|^{\frac{1}{2}-\frac{1}{2 p}}\left\|f_{k}\right\|_{H}+\right. \\
& \left.+\left\|L_{k}(\lambda) u_{1}\right\|_{(H(A), H)_{\frac{1}{2}+\frac{1}{2 p}, p}}+|\lambda|^{\frac{1}{2}-\frac{1}{2 p}}\left\|L_{k}(\lambda) u_{1}\right\|_{H}\right) \leq \\
& \leq C(\varphi, \varepsilon)\left[\sum_{k=1}^{2}\left(\left\|f_{k}\right\|_{(H(A), H)_{\frac{1}{2}+\frac{1}{2 p}, p}}+|\lambda|^{\frac{1}{2}-\frac{1}{2 p}}\left\|f_{k}\right\|_{H}\right)+\right. \\
& +|\lambda|\left\|u_{1}(0)\right\|_{(H(A), H)_{\frac{1}{2}+\frac{1}{2 p}, p}}+\left\|u_{1}^{\prime}(0)\right\|_{(H(A), H)_{\frac{1}{2}+\frac{1}{2 p}, p}}+\left\|B_{1} u_{1}(1)\right\|_{(H(A), H)_{\frac{1}{2}+\frac{1}{2 p}, p}}+ \\
& +|\lambda|\left\|u_{1}(1)\right\|_{(H(A), H)_{\frac{1}{2}+\frac{1}{2 p}, p}}+\left\|u_{1}^{\prime}(1)\right\|_{(H(A), H)_{\frac{1}{2}+\frac{1}{2 p}, p}}+\left\|B_{2} u_{1}(0)\right\|_{(H(A), H)_{\frac{1}{2}+\frac{1}{2 p}, p}}+ \\
& +|\lambda|^{\frac{1}{2}-\frac{1}{2 p}}\left(|\lambda|\left\|u_{1}(0)\right\|_{H}+\left\|u_{1}^{\prime}(0)\right\|_{H}+\left\|B_{1} u_{1}(1)\right\|_{H}+|\lambda|\left\|u_{1}(1)\right\|_{H}+\right. \\
& \left.\left.+\left\|u_{1}^{\prime}(1)\right\|_{H}+\left\|B_{2} u_{1}(0)\right\|_{H}\right)\right] \text {. }
\end{aligned}
$$

By virtue of $\left[13\right.$, theorem 1.7.7/1 ] and the estimate (3.7), for any $x_{0} \in[0,1]$, $s=0,1$,

$$
\left\|u_{1}^{(s)}\left(x_{0}\right)\right\|_{(H(A), H) \frac{s}{2}+\frac{1}{2 p}, p} \leq C\left\|u_{1}\right\|_{W_{p}^{2}((0,1) ; H(A), H)} \leq C(\varphi)\|f\|_{L_{p}((0,1) ; H)} .
$$

By virtue of $[13$, theorem $1.7 .7 / 2]$, for $\mu \in \mathbb{C}, u_{1} \in W_{p}^{2}((0,1) ; H)$, the estimate is satisfied

$$
\begin{gathered}
|\mu|^{2-s}\left\|u_{1}^{(s)}\left(x_{0}\right)\right\|_{H} \leq \\
\leq C\left(|\mu|^{\frac{1}{p}}\left\|u_{1}\right\|_{W_{p}^{2}((0,1) ; H)}+|\mu|^{2+\frac{1}{p}}\left\|u_{1}\right\|_{L_{p}((0,1) ; H)}\right), s=0,1 .
\end{gathered}
$$

Dividing (3.10) by $|\mu|^{\frac{1}{p}}$ and denoting $\lambda=\mu^{2}$, for $\lambda \in \mathbb{C},|\lambda| \rightarrow \infty$, $u_{1} \in W_{p}^{2}((0,1) ; H)$, we have 


$$
|\lambda|^{1-\frac{s}{2}-\frac{1}{2 p}}\left\|u_{1}^{(s)}\left(x_{0}\right)\right\|_{H} \leq C\left(\left\|u_{1}\right\|_{W_{p}^{2}((0,1) ; H)}+|\lambda|\left\|u_{1}\right\|_{L_{p}((0,1) ; H)}\right), s=0,1 .
$$

Then, from (3.7) and (3.11) for $|\arg \lambda| \leq \varphi,|\lambda| \rightarrow \infty$, we have

$$
|\lambda|^{1-\frac{s}{2}-\frac{1}{2 p}}\left\|u_{1}^{(s)}\left(x_{0}\right)\right\|_{H} \leq C(\varphi)\|f\|_{L_{p}((0,1) ; H)}, s=0,1 .
$$

Since the imbedding $(H(A), H)_{\frac{1}{2 p}, p} \subset H$ is continuous, by condition 3 ) of theorem 2.1 and the estimate (3.9), we get

$$
\left\|B_{1} u_{1}(1)\right\|_{H} \leq\left\|u_{1}(1)\right\|_{H} \leq C\left\|u_{1}(1)\right\|_{(H(A), H) \frac{1}{2 p}, p} \leq C(\varphi)\|f\|_{L_{p}((0,1) ; H)} .
$$

By condition 4) of theorem 2.1,

$$
\left\|B_{2} u_{1}(0)\right\|_{H}=\left\|B_{2} A^{-1 / 2} A^{1 / 2} u_{1}(0)\right\|_{H} \leq C\left\|A^{1 / 2} u_{1}(0)\right\|_{H} .
$$

Estimate the norm $\left\|A^{1 / 2} u_{1}(0)\right\|_{H}$. Since $u_{1}(0) \in(H(A), H)_{\frac{1}{2 p}, p}$ and the operator $A^{1 / 2}$ is bounded from $(H(A), H)_{\frac{1}{2 p}, p}$ into $(H(A), H)_{\frac{1}{2}+\frac{1}{2 p}, p}$ (see [12, theorem $1.15 .2 /(\mathrm{e})])$, then $A^{1 / 2} u_{1}(0) \in(H(A), H)_{\frac{1}{2}+\frac{1}{2 p}, p}$. Since the imbedding $(H(A), H)_{\frac{1}{2}+\frac{1}{2 p}, p} \subset H$ is continuous, from (3.9) we have

$$
\begin{gathered}
\left\|A^{1 / 2} u_{1}(0)\right\|_{H} \leq C\left\|A^{1 / 2} u_{1}(0)\right\|_{(H(A), H)_{\frac{1}{2}+\frac{1}{2 p}, p}} \leq \\
\leq C\left\|u_{1}(0)\right\|_{(H(A), H) \frac{1}{2 p}, p} \leq C(\varphi)\|f\|_{L_{p}((0,1) ; H)} .
\end{gathered}
$$

Taking into account (3.15) in (3.14), we get

$$
\left\|B_{2} u_{1}(0)\right\|_{H} \leq C(\varphi)\|f\|_{L_{p}((0,1) ; H)} .
$$

As the imbedding $(H(A), H)_{\frac{1}{2 p}, p} \subset(H(A), H)_{\frac{1}{2}+\frac{1}{2 p}, p}$ is continuous, by virtue of condition 3 ) of theorem 2.1 (see also theorem 1.1) and the estimate (3.9), we have

$$
\begin{gathered}
\left\|B_{1} u_{1}(1)\right\|_{(H(A), H)_{\frac{1}{2}+\frac{1}{2 p}, p}} \leq C\left\|B_{1} u_{1}(1)\right\|_{(H(A), H) \frac{1}{2 p}, p} \leq \\
\leq C\left\|u_{1}(1)\right\|_{(H(A), H) \frac{1}{2 p}, p} \leq C(\varphi)\|f\|_{L_{p}((0,1) ; H)} .
\end{gathered}
$$

By virtue of the estimates (2.16) and (3.9),

$$
\left\|B_{2} u_{1}(0)\right\|_{(H(A), H)_{\frac{1}{2}+\frac{1}{2 p}, p}} \leq C\left\|u_{1}(0)\right\|_{(H(A), H) \frac{1}{2 p}, p} \leq C(\varphi)\|f\|_{L_{p}((0,1) ; H)} .
$$

By the estimates (3.9), (3.12), (3.13), (3.16), (3.17), and (3.18), from (3.8) for $|\arg \lambda| \leq \varphi-\varepsilon,|\lambda| \rightarrow \infty$, we get the estimate (3.3). Theorem 3.1 is proved.

Remark 3.1. Instead of the boundary value problem (3.1), (3.2) in $H$ we can consider a boundary value problem with perturbation operators in the boundary conditions, i.e., the problem

$$
\begin{gathered}
L(\lambda) u:=\lambda u(x)-u^{\prime \prime}(x)+A u(x)=f(x), \quad x \in(0,1), \\
L_{1}(\lambda) u:=\lambda u(0)-\alpha u^{\prime}(0)+B_{1} u(1)+T_{1} u=f_{1}, \\
L_{2}(\lambda) u:=\lambda u(1)+\beta u^{\prime}(1)+B_{2} u(0)+T_{2} u=f_{2},
\end{gathered}
$$


where $T_{k}, k=1,2$, are operators acting from $L_{p}((0,1) ; H), p \in(1, \infty)$ into $H$.

Imposing some conditions on the operators $T_{k}, k=1,2$, we can prove a theorem on solvability of the boundary value problem $(3.19),(3.20)$ by the same way as in papers [4], [6].

\section{Application of the abstract results to elliptic partial differential equations}

In the square $\Omega=[0,1] \times[0,1]$, we consider a boundary value problem for a second order elliptic partial differential equation with a complex parameter in the equation and in the boundary conditions

$$
\begin{gathered}
L(\lambda) u:=\lambda u(x, y)-D_{x}^{2} u(x, y)- \\
-D_{y}\left(a(y) D_{y} u(x, y)\right)=f(x, y),(x, y) \in(0,1) \times(0,1), \\
L_{1}(\lambda) u:=\lambda u(0, y)-\alpha D_{x} u(0, y)+b_{1}(y) u(1, y)+\int_{0}^{1} b_{1}(t, y) u(1, t) d t=f_{1}(y), y \in[0,1], \\
L_{2}(\lambda) u:=\lambda u(1, y)+\beta D_{x} u(1, y)+b_{2}(y) D_{y} u(0, y)+c(y) u(0, g(y))+ \\
+\int_{0}^{1}\left(b_{2}(t, y) D_{t} u(0, y)+c(t, y) u(0, h(y))\right) d t=f_{2}(y), \quad y \in[0,1] \\
L_{3} u:=u(x, 0)=0, \quad x \in[0,1] \\
L_{4} u:=u(x, 1)=0, x \in[0,1] .
\end{gathered}
$$

Here $\lambda$ is a complex parameter; $\alpha, \beta$ are some complex numbers; $b_{1}(y), b_{2}(y), c(y)$, $h(y), g(y), b_{1}(x, y), b_{2}(x, y), c(x, y)$ are continuous functions; $D_{x}=\frac{\partial}{\partial x}, \quad D_{y}=\frac{\partial}{\partial y}$.

Denote the interpolation space of Sobolev spaces by

$$
B_{p, q}^{s}(0,1):=\left(W_{p}^{s_{0}}(0,1), W_{p}^{s_{1}}(0,1)\right)_{\theta, q},
$$

where $0 \leq s_{0}, s_{1}$ are integers, $0<\theta<1,1<q<\infty, 1<p<\infty$ and $s=(1-\theta) s_{0}+\theta s_{1}$. In particular, $W_{p}^{s}(0,1):=B_{p, p}^{s}(0,1):=\left(W_{p}^{s_{0}}(0,1), W_{p}^{s_{1}}(0,1)\right)_{\theta, p}$, if $0<s \neq$ an integer.

Theorem 4.1. Let the following conditions be fulfilled:

1) $\alpha, \beta$ are some non-zero complex numbers with $|\arg \alpha| \leq \frac{\pi-\varphi}{2}$ and $|\arg \beta| \leq$ $\frac{\pi-\varphi}{2}$, for some $\varphi \in(0, \pi) ; a(y) \in C^{1}[0,1], a(y)>0$, for $y \in[0,1]$;

2) $b_{1}(y) \in C^{2}[0,1], \quad b_{1}(0)=b_{1}(1)=b_{1}^{\prime}(0)=b_{1}^{\prime}(1)=0 ; \quad b_{1}(x, y) \in C^{0,2}(\Omega)$, where $C^{0,2}(\Omega)$ is the space of continuous functions in the square $\Omega$, which have continuous derivatives $\frac{\partial b_{1}(x, y)}{\partial y}, \frac{\partial^{2} b_{1}(x, y)}{\partial y^{2}}$, and $b_{1}(x, 0)=b_{1}(x, 1)=\frac{\partial b_{1}(x, 0)}{\partial y}=$ $\frac{\partial b_{1}(x, 1)}{\partial y}=0 ; b_{2}(y) \in C^{1}[0,1], b_{2}(0)=b_{2}(1)=0 ; c(y), g(y), h(y) \in C^{1}[0,1] ;$ $g(y)$ and $h(y)$ map $[0,1]$ into itself, $c(0)=c(1)=0 ; b_{2}(t, y), c(t, y) \in C^{1}(\Omega)$ and $b_{2}(t, 0)=b_{2}(t, 1)=c(t, 0)=c(t, 1)=0$, for $t \in[0,1]$.

Then, the operator $\mathbb{L}(\lambda): u \rightarrow \mathbb{L}(\lambda) u:=\left(L(\lambda) u, L_{1}(\lambda) u, L_{2}(\lambda) u\right)$ for suffciently large $\lambda$ from the angle $|\arg \lambda| \leq \varphi-\varepsilon$, where $\varepsilon>0$ is a sufficiently small number, is an isomorphism from $W_{p}^{2}\left((0,1) ; W_{2}^{2}(0,1), L_{2}(0,1), u(0)=u(1)=0\right)$ 
into $L_{p}\left((0,1) ; L_{2}(0,1)\right)+B_{2, p, *}^{1-\frac{1}{p}}(0,1)+B_{2, p, *}^{1-\frac{1}{p}}(0,1)$ and for the solution $u(x, y)$ of the problem (4.1)-(4.3), $u(0, y) \in W_{2}^{1}(0,1)$ and the following estimate is valid

$$
\begin{gathered}
|\lambda|\|u(x, y)\|_{L_{p}\left((0,1) ; L_{2}(0,1)\right)}+\left\|D_{x}^{2} u(x, y)\right\|_{L_{p}\left((0,1) ; L_{2}(0,1)\right)}+\left\|D_{y}^{2} u(x, y)\right\|_{L_{p}\left((0,1) ; L_{2}(0,1)\right)} \\
\leq C(\varphi, \varepsilon)\left[|\lambda|\|f(x, y)\|_{L_{p}\left((0,1) ; L_{2}(0,1)\right)}+\sum_{k=1}^{2}\left(\left\|f_{k}(y)\right\|_{B_{2, p}^{1-\frac{1}{p}}(0,1)}+\right.\right. \\
\left.\left.+|\lambda|^{\frac{1}{2}-\frac{1}{2 p}}\left\|f_{k}(y)\right\|_{L_{2}(0,1)}\right)\right]
\end{gathered}
$$

where

$$
B_{2, p, *}^{1-\frac{1}{p}}(0,1)=\left\{\begin{array}{l}
B_{2, p}^{1-\frac{1}{p}}(0,1), \quad 1<p<2, \\
W_{2}^{1 / 2}\left((0,1) ; \int_{0}^{1}(\min \{x, 1-x\})^{-1}|u(x)|^{2} d x<\infty\right), \quad p=2, \\
B_{2, p}^{1-\frac{1}{p}}((0,1), u(0)=u(1)=0), p>2 .
\end{array}\right.
$$

Proof. In the space $H:=L_{2}(0,1)$, we consider the operators $A, B_{1}, B_{2}$ given by the following equalities

$$
\begin{aligned}
D(A):= & W_{2}^{2}((0,1) ; u(0)=u(1)=0), \quad A u:=-\left(a(y) u^{\prime}(y)\right)^{\prime}, \\
D\left(B_{1}\right):= & L_{2}(0,1), \quad B_{1} u:=b_{1}(y) u(y)+\int_{0}^{1} b_{1}(t, y) u(t) d t, \\
D\left(B_{2}\right):= & W_{2}^{1}(0,1), \quad B_{2} u:=b_{2}(y) u^{\prime}(y)+c(y) u(g(y))+ \\
& \int_{0}^{1}\left(b_{2}(t, y) u^{\prime}(t)+c(t, y) u(h(t))\right) d t .
\end{aligned}
$$

Then, the problem (4.1)-(4.3) can be rewritten in the operator form (3.1), (3.2) and one can apply theorem 3.1. Verify the conditions of theorem 3.1 for the all considered operators (4.4)-(4.6).

From condition 1) of theorem 4.1, if follows that the operator $A$ determined by the equalities in (4.4), is self-adjoint and positive definite in the space $L_{2}(0,1)$. Therefore, condition 1) of theorem 2.1 is fulfilled for any $0<\varphi<\pi$.

It is easily shown that the operator $B_{1}$ determined by the equalities in (4.5), subject to condition 2) of theorem 4.1, is bounded in the spaces $L_{2}(0,1)$ and $W_{2}^{2}(0,1)$. Hence, condition 3$)$ of theorem 2.1 is fulfilled, too.

Condition 4) of theorem 2.1 for the operator $B_{2}$ determined by the equalities in (4.6), is verified in the same way as in the proof of theorem 7.1 from [6].

Now, we give an explicit form of the interpolation space $(H(A), H)_{\frac{1}{2}+\frac{1}{2 p}, p}$. By theorem 4.3.3 from [12],

$$
(H(A), H)_{\frac{1}{2}+\frac{1}{2 p}, p}=\left(W_{2}^{2}((0,1) ; u(0)=u(1)=0), L_{2}(0,1)\right)_{\frac{1}{2}+\frac{1}{2 p}, p}=
$$




$$
=\left\{\begin{array}{l}
B_{2, p}^{1-\frac{1}{p}}(0,1), \quad 1<p<2, \\
W_{2}^{1 / 2}\left((0,1) ; \int_{0}^{1}(\min \{x, 1-x\})^{-1}|u(x)|^{2} d x<\infty\right), \quad p=2, \\
B_{2, p}^{1-\frac{1}{p}}((0,1), u(0)=u(1)=0), \quad p>2,
\end{array}\right.
$$

i.e, $(H(A), H)_{\frac{1}{2}+\frac{1}{2 p}, p}=B_{2, p, *}^{1-\frac{1}{p}}(0,1)$.

\section{References}

[1] A.Aibeche, A.Favini, Ch.Mezoued, Deficient coerciveness estimate for an abstract differential equation with a parameter dependent boundary conditions, Boll Unione Mat. Ital. Sez. B Artic. Ric. Mat. (8)10 (2007), no 3, 535-547.

[2] B.A.Aliev, A boundary value problem for a second order elliptic differential -operator equation with a spectral parameter and operator boundary conditions, Proc. Inst. Math. Mech. Natl. Acad. Sci. Azerb. 32 (2010), 21-46.

[3] B.A.Aliev, N.K.Kurbanova and Ya.Yakubov, On a boundary value problem for second order elliptic differential- operator equations with quadratic spectral parameter, Ukrainian Math. J. 69 (2017), no 6, 857-875.

[4] B.A.Aliev, N.K.Kurbanova, Ya. Yakubov, Solvability of a boundary value problem for second - order elliptic differential operator equations with a spectral parameter in the equation and in the boundary conditions, Differ. Equ. 54 (2018), no.1, 67-85.

[5] B.A.Aliev, Ya.Yakubov, Elliptic differential-operator problems with a spectral parameter in both the equation and boundary-operator conditions, Adv. Differential Equations, 11 (2006), no 10, 1081-1110. (Erratum in 12 (2007), no.9, 1079).

[6] B.A.Aliev, Ya.Yakubov, Second order elliptic differential operator equations with unbounded operator boundary conditions in UMD Banach spaces, Integral Equations Operator Theory 69 (2011), no.2, 269-300.

[7] N.M.Aslanova, M.Bayramoglu, K.M.Aslanov, On one class eigenvalue problem with eigenvalue parameter in the boundary condition at one end-point, Filomat 32 (2018), no.19, 6667-6674.

[8] M.Denche, Abstract differential equation with a spectral parameter in the boundary conditions, Results Math. 35 (1999), no.3-4, 216-227.

[9] R.Denk, M.Hieber, J. Prus, R-Boundedness, Fourier multipliers and problems of elliptic and parabolic type, Mem. Amer. Math. Soc., 166 (2003), no. 788.

[10] A.Favini, V.Shakhmurov, Ya.Yakubov, Regular boundary value problems for complete second order elliptic differential-operator equations in UMD Bahach spaces, Semigroup Forum 79 (2009), no.1, 22-54.

[11] M.A. Rybak, On asymptotic distribution of eigenvalues of certain boundary value problems for the operator Sturm-Liouville equation, Ukrain. Mat. Zh. 32 (1980), no.2, 248-252.

[12] H.Tribel, Interpolation theory. Functional spaces. Differential operators. "Mir", Moscow, 1980.

[13] S.Yakubov, Ya. Yakubov, Differential-operator equations. Ordinary and Partial Differential Equations. Chapman and Hall/CRC, Boca Raton, FL, 2000.

[14] Ya.Yakubov, Elliptic differential-operator problems with the spectral parameter in both the equation and boundary conditions and the corresponding abstract parabolic initial boundary value problems, New Prospects in Direct, Inverse and Control Problems for Evolution Equations, 437-471, Springer INdAM Ser., 10 Springer, Cham, 2014 . 
Bahram A. Aliev

Institute of Mathematics and Mechanics of NAS of Azerbaijan, Baku, Azerbaijan, Azerbaijan State Pedagogical University, Baku, Azerbaijan

E-mail address: aliyevbakhram@yandex.ru

Vugar Z. Kerimov

Azerbaijan State Pedagogical University, Baku, Azerbaijan

E-mail address: vugarkerimli@mail.ru

Yakov S. Yakubov

Tel Aviv University, Tel Aviv, Israel

E-mail address: yakubov@tauex.tau.ac.il

Received: February 4, 2021; Revised: July 21, 2021; Accepted: August 3, 2021 